\title{
Identification of Flagellated or Fimbriated Bacterial Cells using Digital Image Processing Techniques
}

\author{
P.S. Hiremath \\ Dept. of Computer Science \\ Gulbarga University \\ Gulbarga, Karnataka, India
}

\author{
Parashuram Bannigidad \\ Dept. of Computer Science \\ Govt. Degree College, \\ Gulbarga, Karnataka, India
}

\author{
Soumyashree S.Yelgond \\ Dept. of Computer Science \\ Gulbarga University \\ Gulbarga, Karnataka, India
}

\begin{abstract}
The objective of the present study is to develop an automatic tool to characterize the morphology of bacterial cells in digital microscopic cell images. Geometric shape features are used to identify the morphological characteristics, namely, flagella and fimbriae or pili of bacterial cells. The current methods rely on the subjective reading of cell profiles by a human expert based on the various manual staining methods for visualization of these characteristics. In this paper, an automatic method is proposed for bacterial cell characterization based on their morphological characteristics by segmenting digital bacterial cell images and extracting geometric shape features that define cell morphology. The experimental results are compared with the manual results obtained by the microbiology expert and, thus, demonstrate the efficacy of the proposed method.
\end{abstract}

\section{Keywords}

Bacterial cell image analysis, flagellum, fimbriae, bacterial cell morphology, digital image analysis, edge detection.

\section{INTRODUCTION}

Today, there are numerous types of bacteria in the world, most bacteria are useful. Some are even necessary to life. Others are put to work for a specific purpose. Bacteria play an important role in the digestive processes of animals. There are several bacteria in the human intestine. As the bacteria eat, they break down foods. At the same time they make certain vitamins, which the body then consumes. The bacteria inside the stomach of grass-eating animals can break down cellulose, the stiff wall of green plant cells. By enabling cows to digest grasses, bacteria play a role in the production of milk. Bacteria, often 'Lactobacillus' in combination with yeasts and molds, have been used for thousands of years in the preparation of fermented foods such as cheese, pickles, soy sauce, sauerkraut, vinegar, wine and yoghurt. The ability of bacteria to degrade a variety of organic compounds is remarkable and is used in waste processing and bioremediation. Bacteria capable of digesting the hydrocarbons in petroleum are often used to clean up oil spills. Bacteria are also used for the bioremediation of industrial toxic wastes. In the chemical industry, bacteria are most important in the production of enantiomerically pure chemicals for usage in pharmaceuticals or agrichemicals.

Bacteria cause the decay of dead plants and animals, both on land and in water. Without such bacteria, the earth would have been covered with material from dead organisms. Bacteria can also be used in place of pesticides in the biological pest control. It commonly involves Bacillus thuringiensis (also called BT), a Gram-positive, soil dwelling bacterium. Because of their specificity, these pesticides are regarded as environment-friendly, with little or no effect on humans, wildlife, pollinators and most other beneficial insects
[15].Surface structures originate outside the cell membrane, sometimes being attached to it, and extend into the environment. Important structures include flagella, pili or fimbriae, cell wall and glycocaylyx [14]. The schematic diagram of bacterial cell structure is shown in the Fig.1.The bacteria possess the morphological structures for the purpose of performing some physiological functions, e.g. flagella for motility and fimbriae for capturing the food in the surrounding of a cell and helps in adhesion to other cells and surfaces.

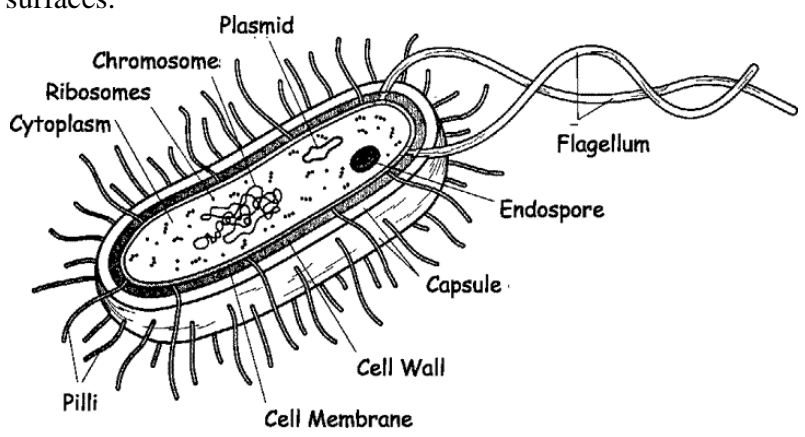

Fig. 1 The schematic diagram of bacterial cell structure.

In this paper, the study is concerned with the two morphological structures, namely, flagella and fimbriae of bacterial cells, which are described below:

\section{Flagellum:}

Flagella are responsible for motility in most bacteria. There is a loose correlation between cell shape the presence of flagella. Almost all spirillum, half of all rods, and rarely cocci are motile via flagella. A spherical shape would probably cause much more drag on the cell than a rod. To be motile, it has been a streamlined rod. Typically microbes that live in aqueous environments will continually move around looking for nutrients. Sometimes this movement is random, but in other cases it is directed toward or away from something. In other words, bacteria are capable of showing simple behavior that depends upon various stimuli. It is possible to watch living bacteria swim around using the phase microscope. In many cases, this motility is due to flagella. This is a semisolid medium that will hold non-motile bacteria in place, but motile microbes can swim through it. The presence of turbidity throughout the tube is a positive test for the presence of motility.

Flagella are responsible for motility in most bacteria and causes urinary tract infections. There is a weak correlation between cell shape the presence of flagella. Almost all spirillum, half of all rods, and rarely cocci are motile via 
flagella. The coccus morphology and motility are mutually exclusive. The structure of flagella can be thought of as little semi-rigid whips that are free at one end and attached to a cell at the other. The diameter of a flagellum is thin, $20 \mathrm{~nm}$, and long with some having a length 10 times the diameter of cell. Due to their small diameter, flagella cannot be seen in the light microscope unless a special stain is applied. Bacteria can have one or more flagella arranged in clumps or spread all over the cell.

\section{Fimbriae:}

Fimbriae are structurally similar to flagella and are composed of one or a few proteins arranged in a helical fashion. Each protein subunit assembles on the growing structure at the tip, as is the case with flagella. There are a number of genes necessary for the successful construction of pili and their products might perform functions such as moving the structural proteins across the membrane, methylating the structural proteins or retracting the pilus. The same is generally true for fimbriae. Fimbriae are found on many bacteria and are shorter and straighter than flagella and are more numerous. Not all bacteria synthesize them. Fimbriae do not function in motility, but are thought to be important in attachment to surfaces. Some microbes attach to hosts by fimbriae, and successful colonization of many surfaces is totally dependent upon the ability to make fimbriae [14].

The complete atomic model of the bacterial flagellar filament by electron cryomicroscopy has been carried out by Koji et al. [1]. The real-time imaging of fluorescent flagellar filaments has been done by Linda et al. [2]. The bacterial growth and motility in sub-micron constrictions has been investigated by Jaan et al. [3]. Hiremath and Parashuram [4] have investigated the automatic Gram-staining characterization of digital microscopic bacterial cell images using color and cell wall properties. A simple image analysis algorithm for evaluation of extended filaments length based on the enhanced digitized image using statistical analysis is proposed [7]. GSI of bacterial and archaeal cells in the natural microbial communities of slightly and extremely saline environments has been proposed [8]. Digital image analysis of actinomycetes colonies as a potential aid for rapid taxonomic identification has been investigated [9]. Characterization of PHB storage in activated sludge extended filamentous bacteria by automated colour image analysis has been examined [10].

In this paper, the objective is to propose a method for automatic identification of flagellated or fimbriated bacterial cells in digital microscopic images using geometric shape features that characterize the different morphology of bacterial cells. The experimental results are compared with the manual results obtained by microbiology expert and demonstrate the efficacy of the proposed method.

\section{MATERIALS AND METHODS}

The strains were inoculated in broth media and incubated overnight at $37^{\circ} \mathrm{C}$ for $18 \mathrm{hrs}$ in agitation rotor. The bacterial cells from each culture were recovered by centrifugation at $6,000 \mathrm{rev} / \mathrm{min}$ and the cells were washed twice with potassium phosphate buffer $(50 \mathrm{mM}, \mathrm{pH} 7.0)$. Bacterial cells were then fixed by immersion in $2.5 \%$ glutaraldehyde in potassium phosphate buffer $(50 \mathrm{mM}, \mathrm{pH} 7)$ overnight at $40 \mathrm{C}$. Then the specimens were washed twice with buffer and dehydrated by an ethanol series (v/v) ranging from $30,40,50,60,70,80,90$ to $100 \%$ and stored in $100 \%$ ethanol. For SEM, the specimens were dried to critical point, coated with gold and examined with an S-200C scanning electron microscope. The cell volumes and surface area were directly measured from Scanning Electronic Microscopy (SEM) photograph. The digital images of cells on the slides are captured by a digital camera interfaced with a PC and then stored in the disk memory for further processing and image analysis. For the purpose of experimentation, a datasets containing the 300 color images of bacterial cells(non-overlapping) with different characteristics, namely, flagellated and fimbriated, is prepared.

\section{PROPOSED METHOD}

The purpose of the automated image analysis of digital bacterial cell image is to identify the morphology of bacterial cell, whether it is flagellated or fimbriated, based on their geometric shape features.

Out of many geometric features used by various authors in the literature[5][6][13], it is observed that there are seven geometric shape features, namely, elongated, circularity, eccentricity, tortuosity, length-width ratio, relative convex area and relative convex perimeter, which provide better classification results. Hence, in the proposed method, these seven features are used, which are defined as given below:

Elongated(x1): It is the ratio of longer side to shorter side of the bounding rectangle (Elongated $=$ Length $/$ Width).

Circularity(x2): It is to measure irregularity of circular objects. Circularity $=4 \pi($ Area $) /$ perimeter 2

Eccentricity $(x 3)$ : It is the ratio between the lengths of the short axis to the long axis(Eccentricity $=$ axis length short / axis length long). The value of eccentricity is between 0 and 1 . Eccentricity is also called ellipticity with respect to minor axis and major axis of the ellipse.

Tortuosity $(x 4)$ : It is the ratio of contour length (perimeter) to the maximum linear length (MajorAxis Length) between any two points on the contour $($ Tortuosity $=$ Major axis $/$ perimeter $)$

Length-width ratio ( $L W$ ratio) $(x 5)$ : It is the ratio of the length of longest chord (Length) of the shape to the longest chord perpendicular to it (Width). (Length-width ratio $=$ Major axis $/$ minor axis).

Relative convex area $(\mathrm{x} 6)$ : It is the ratio of convex area to area (Relative convex area $=$ convex area $/$ area).

Relative convex perimeter $(\mathrm{x} 7)$ : It is the ratio of convex perimeter to perimeter (Relative convex perimeter $=$ convex perimeter $/$ perimeter $)$.

The proposed method comprises the following steps:

- Preprocessing using morphological operations.

- Segmentation using Canny's edge method.

- Geometric feature extraction. 
- Classification into flagellated and fimbriated cells.
The block diagram of the proposed method is shown in the Fig.2.

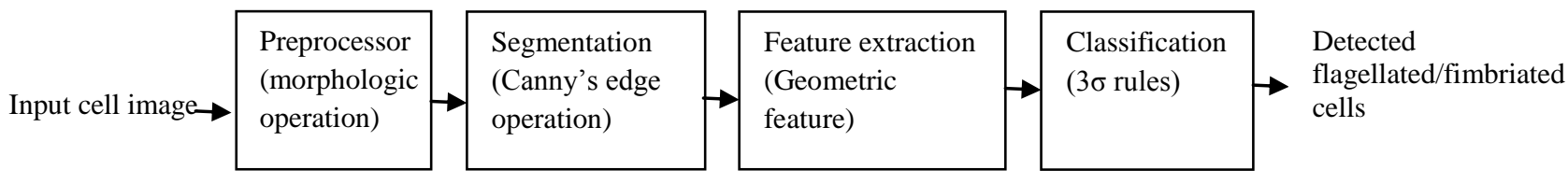

Fig.2 The block diagram of the proposed method
The bacterial cell images generally contain noise, small debris and artifacts depending on the different staining methods. To remove this debris, preprocessed the image by applying morphological operations. This stage is of high importance in achieving good results in segmentation and further process. The gray scale image of cells is segmented using the canny's edge method, which yields edge of the image. After labeling the segmented image, the geometric features, $x_{i}, i=1,2, \ldots 7$, are extracted for each labeled segment. These features are used as a basis for the cell characteristics. Using the training set of images (with known cell classification), for each feature $x_{i}, i=1,2, \ldots 7$, of $\mathrm{k}^{\text {th }}$ cell type, compute the mean $x_{i}^{-k}$ and standard deviation $\sigma_{i}^{k}$ of the sampling distribution of the feature values and store them as knowledge base. In the testing phase, for a given test image, feature values $x_{i}^{(t e s t)}$ of the segmented regions (cells) are computed and then cell classification is done using the $3 \sigma$ rule, namely: For a segmented region in the test image, if the feature values $x_{i}^{\text {(test) }}$ lie in the interval $x_{i}^{-k} \pm 3 \sigma_{i}^{k,} i=1,2, \ldots 7$, then the region is a cell of type $\mathrm{k}$. The $\mathrm{k}=1,2$ correspond to flagellated and fimbriated cell types, respectively.

The proposed method for the automatic identification and classification of bacterial cell characteristics in digital microscopic images using geometric shape features that characterize the different morphology of bacterial cells is given in the Algorithms 1 and 2:

Training phase:

Algorithm 1: Extraction of features for knowledge base

Step 1:Input bacterial cell image (RGB color training image).

Step 2: Convert the RGB image into gray scale image.

Step 3:Perform preprocessing using morphological operations.

Step 4: Segment the resulting image of Step 3 using canny's edge method.

Step 5: Perform labeling the segmented image.

Step 6: For each labeled segment, compute geometric shape features, $x_{i}, i=1,2, \ldots 7$, (i.e. elongated, circularity, eccentricity, tortuosity, LW ratio, relative convex area and relative convex perimeter) for each cell type $\mathrm{k}$. The $\mathrm{k}=1,2$ correspond to flagellated and fimbriated cells, respectively.

Step 7: Repeat the Steps 1 to 6 for all the training images.

Step 8: Compute mean $x_{i}^{-k}$ and standard deviation $\sigma_{i}^{k}$ of the sampling distribution of the feature values for each cell type $\mathrm{k}$ and store them as knowledge base.

\section{Classification (Testing) phase:}

Algorithm 2: Classification of bacterial cell morphology

Step 1: Input bacterial cell image (RGB color training image).

Step 2: Convert the RGB image into gray scale image.

Step 3:Perform preprocessing using morphological operation.

Step 4: Segment the resulting image of Step 3 using canny's edge method.
Step 5: Perform labeling the segmented image.

Step 6: For each labeled segment, compute geometric shape features $x_{i}, i=1,2, \ldots 7$, (i.e. elongated, circularity, eccentricity, tortuosity, LW ratio, relative convex area and relative convex perimeter) and store these features as $x_{i}^{(t e s t)}$.

Step 7: Apply $3 \sigma$ rule for classification of the bacterial cells: A segmented region is of cell type $\mathrm{k}$, if its features $x_{i}^{(t e s t)}$ lie in the interval $x_{i}^{-k} \pm 3 \sigma_{i}^{k,} i=1,2, \ldots 7$, . The $\mathrm{k}=1$, 2 correspond to flagellated and fimbriated cell type, respectively.

Step 8: Repeat the Steps 6 and 7 for all labeled segments and output the classification of identified cells.

\section{EXPERIMENTAL RESULTS AND DISCUSSIONS}

For the purpose of experimentation, 300 color digital bacterial cell images containing different characteristics of bacterial cells (non-overlapping) namely, flagellated and fimbriated spiral are considered (as described in the section 2). The implementation is done on a Pentium Core 2 Duo @ 2.83 GHz machine using MATLAB 7.9. In the training phase, each input color image of bacterial cell (Fig.3(a)) is converted into gray scale image, and the morphological operations such as erosion, reconstruction and dilation are applied $[12,13]$. The resulting image is segmented using canny's edge method to obtain segmented binary image (Fig.3 (b)). The segmented image is labeled and for each segmented region (known cells), the geometric features are computed. The Table I presents the geometric feature values computed for the segmented cell regions of the image in Fig. 3(b) and 1(d).

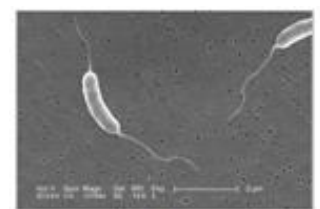

(a)

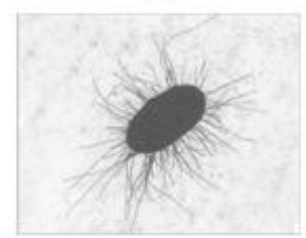

(c)

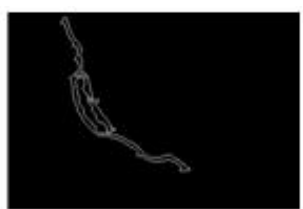

(b)

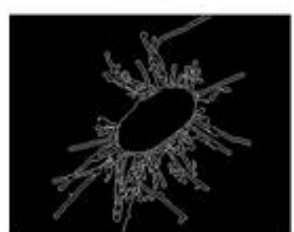

(d)
Fig. 3 (a) Microscopic flagellated cell image, (b) Segmented image of image in (a), (c) Microscopic fimbriated cell image, (d) Segmented image of image in (c). 
Table I

The geometric feature values of the cell regions of the image in fig. $3(\mathrm{~b})$ and $(\mathrm{d})$

\begin{tabular}{|l|c|c|}
\hline \multirow{2}{*}{\begin{tabular}{c}
\multirow{2}{*}{$\begin{array}{c}\text { Geometric cell } \\
\text { features }\end{array}$} \\
\cline { 2 - 3 }
\end{tabular}} & $\begin{array}{c}|c| \\
\text { Flagellated } \\
\text { (Fig.3(a)) }\end{array}$ & $\begin{array}{c}\text { Fimbriated } \\
\text { (Fig.3(d)) }\end{array}$ \\
\hline Elongated & 1.2025 & 1.4016 \\
\hline Circularity & 0.0160 & 0.0024 \\
\hline Eccentricity & 0.9609 & 0.7090 \\
\hline Tortousity & 0.3304 & 0.0555 \\
\hline LW ratio & 3.6129 & 1.4180 \\
\hline Relconvarea & 0.2744 & 0.0136 \\
\hline Relconvperim & 0.0663 & 0.0057 \\
\hline
\end{tabular}

The mean and standard deviation of the sampling distribution of these features obtained from the training images are stored in the knowledge base of the cells: flagelatted and fimbriated, as shown in Table II. In the testing phase, for a test image, the feature extraction algorithm is applied and the test feature values $x_{i}^{\text {(test) }}$ for each segmented region are used for classification using $3 \sigma$ rule. The classification results are given in the Table III. For testing images, the $3 \sigma$ classifier has yielded an accuracy of $84.75 \%$ to $92.5 \%$. The sample misclassification results are shown in the Fig.4. In the Fig.4 (d), it is observed that poor quality of segmentation has led to the misclassification, whereas in Fig.4 (b), the $3 \sigma$ classifier is not good enough to classify it correctly. Hence,there is a need to improve both segmentation and classification methods to obtain better results.

Table II

Mean and standard deviation of geometric feature values of flagelatted and fimbriated bacterial cells

\begin{tabular}{|l|c|c|c|c|}
\hline \multirow{2}{*}{$\begin{array}{c}\text { Geometric } \\
\text { features }\end{array}$} & \multicolumn{2}{|c|}{ Flagellated cell } & \multicolumn{2}{c|}{ Fimbriated cell } \\
\cline { 2 - 5 } & Mean & Std & Mean & Std \\
\hline Elongated & 1.56137 & 0.4566 & 1.2060 & 0.1534 \\
\hline Circularity & 0.01018 & 0.0037 & 0.0029 & 0.0004 \\
\hline Eccentricity & 0.85817 & 0.1435 & 0.6551 & 0.1027 \\
\hline Tortousity & 0.26886 & 0.0697 & 0.0605 & 0.0081 \\
\hline LW ratio & 2.52099 & 0.8801 & 1.3609 & 0.1559 \\
\hline Relconvarea & 0.13408 & 0.0961 & 0.0187 & 0.0036 \\
\hline Relconvperim & 0.03551 & 0.0171 & 0.0074 & 0.0011 \\
\hline
\end{tabular}

Table III

Classification accuracy

\begin{tabular}{|c|c|c|}
\hline $\begin{array}{c}\text { Bacterial } \\
\text { characteristics }\end{array}$ & $\begin{array}{c}\text { No. of cells in test } \\
\text { images }\end{array}$ & $\begin{array}{c}\text { Classification } \\
\text { accuracy }(\%)\end{array}$ \\
\hline Flagellated & 350 & 92.5 \\
\hline Fimbriated & 300 & 84.75 \\
\hline
\end{tabular}

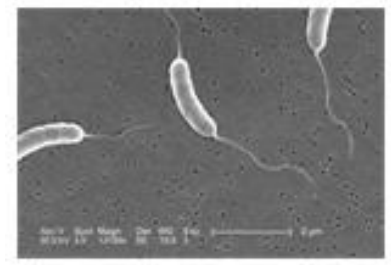

(a)

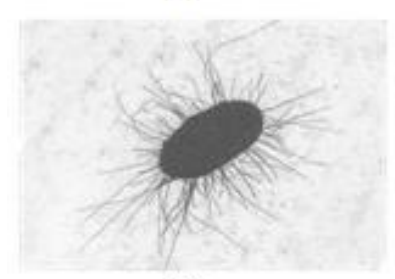

(c)

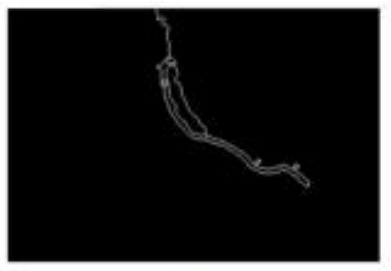

(b)

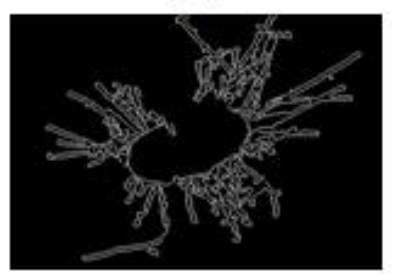

(d)
Fig.4 Sample misclassification results (a) original flagellated cell image, (b) Segmented image of image in (a),(c) original fimbriated cell image, (d) Segmented image of image in (c).

\section{CONCLUSION}

In this paper, an automated method is proposed for cell identification and classification by segmenting digital microscopic bacterial cell morphology images and extracting geometric features of bacterial cell characteristics. Geometric shape features are used to identify the morphological characteristics, namely, flagella and fimbriae or pili of bacterial cells. The experimental results are compared with the manual results obtained by expert. The proposed method is computationally less expensive and yet yields comparable classification rates. The $3 \sigma$ classifier has yielded an accuracy of $84.75 \%$ to $92.5 \%$ for different bacterial cell characteristics. Further, the results can be improved by using better segmentation and classification techniques, which will be done in the future work.

\section{ACKNOWLEDGEMENTS}

The authors are grateful to the referees for their valuable comments and suggestions. Further, the authors are indebted to Dr. A. Dayanand, Professor of Microbiology, Gulbarga University, Gulbarga, for providing bacterial cell images and manual results by visual inspection. This research work is financially supported by Vision Group on Science and Technology, Department of Information Technology, Biotechnology and Science and Technology, Govt. of Karnataka. Bangalore, India.

\section{REFERENCES}

[1] Koji Yonekura, Saori Maki-Yonekura and Keiichi Namba, Complete atomic model of the bacterial flagellar filament by electron cryomicroscopy, NATURE, Vol. 424, 2003, pp. 643-650.

[2] Linda Turner, William S. Ryu, and Howard C. Berg, Real-Time Imaging of Fluorescent Flagellar Filaments, Journal of Bacteriology, Vol. 182, No. 10, 2000, pp. 2793-2801.

[3] Jaan Mannik, Rosalie Driessen, Peter Galajda, Juan E. Keymer, and Cees Dekker1, Bacterial growth and motility in sub-micron constrictions, PNAS, Vol. 106, No. 35, 2009, pp. 14861-14866. 
[4] P.S. Hiremath and Parashuram Bannigidad, Automated Gram-staining characterisation of bacterial cells using colour and cell wall properties, Int. J. Biomedical Engineering and Technology, Vol. 7, No. 3, 2011, pp. 257-265.

[5] Carolina Wahlby, et al., "Algorithms for cytoplasm segmentation of fluorescence labeled cells", Analytical Cellular Pathology, 24, 101-111 (2002).

[6] Venkataraman, S., et al., "Automated image analysis of atomic microscopy images of rotavirus particles", Ultramicroscopy, Elsevier, Vol.106, 829-837 (2006).

[7] Kima, Y.J., Choib, Y.G. and Chunga, T.H. (2008) 'A simple image analysis algorithm for evaluation of extended filaments length based on the enhanced digitized image', Journal of Environmental Science and Health Part A, Vol. 43, pp.1489-1494.

[8] Saida, H., Kamekura, M., El-Sayed, W.S.M., AbuShady, M., Abe, Y., Yamaguchi, T., Yang, P., Maekawa, T. and Seki, H. (2001) 'Gram Stain Index (GSI) of bacterial and archaeal cells in the natural microbial communities of slightly and extremely saline environments', Journal of Oceanography, Vol. 57, pp.109-113.
[9] Velho-Pereira, S. and Kamat, N. (2010) 'Digital image analysis of actinomycetes colonies as a potential aid for rapid taxonomic identification', Nature Preceding: doi:10.1038/npre. 2010.4209.1, pp.1-13.

[10] Pandolfi, D., Pons, M-N. and da Motta, M. (2007) 'Characterization of PHB storage in activated sludge extended filamentous bacteria by automated colour image analysis', Biotechnol. Lett., Vol. 29, pp.12631269.

[11] Rafael C. Gonzalez and Richard E. Woods (2002). Digital Image Processing, Pearson Education Asia, India.

[12] John C. Russ, The Image Processing Hand Book, (2007), $5^{\text {th }}$ Ed. CRC Press, New Jersey.

[13] Evangelia Micheli-Tzanakou "Supervised and Unsupervised Pattern Recognition - Feature Extraction and Computational Intelligence", (2000) CRC Press LLC, Florida.

[14] Jeffrey C. Pommerville (2010) Alcamo's Fundamentals of Microbiology Body systems edition, Jones and Bartlett Publishers, USA.

[15] Dubey and Maheshwari, "The textbook of Microbiology", Revised $1^{\text {st }}$ Ed, 2010. 